\title{
Flexoelectric polarisation effects in nematic liquid crystal phase gratings.
}

\author{
C.L. Trabi ${ }^{\mathrm{a}}$, A.A.T. Smith ${ }^{\mathrm{b}}$, N.J. Mottram ${ }^{\mathrm{b}}$, C.V. Brown ${ }^{\mathrm{a}}$ \\ ${ }^{a}$ School of Science and Technology, Nottingham Trent University, \\ Erasmus Darwin Building, Clifton Lane, Clifton, Nottingham, NG11 8NS; \\ ${ }^{b}$ Department of Mathematics, University of Strathclyde, Livingstone Tower, \\ 26 Richmond Street, Glasgow, G1 1XH, United Kingdom.
}

\begin{abstract}
Nematic phase gratings have been studied in which a planar nematic layer of thickness $17.2 \mu \mathrm{m}$ is sandwiched between two glass substrates coated with an alignment polymer. The upper substrate is a continuous earth plane and the lower substrate has a patterned electrode of interdigitated stripes (electrodes and gaps are both $40 \mu$ mide). Reorientation of the nematic liquid crystal occurs in response to d.c. electric fields applied between the interdigitated electrodes. These nematic reorientation regions have been used to investigate the influence of the flexoelectric polarisation in the nematic liquid crystal by observing the resultant (i) movement of tilt fringes in a Mach-Zehnder interferometer, and (ii) optical diffraction patterns. In the Mach-Zehnder interferometer the periodic variation of the refractive index resulting from the periodic distortion profile is measured directly from the displacement of the tilt fringes. The asymmetry in the response to positive and negative polarities of the d.c. voltage for both measurement techniques is directly related to the sum of the flexoelectric coefficients, $e_{1}+e_{3}$.
\end{abstract}

Keywords: Phase grating, nematic liquid crystal, diffractive optical element.

*carl.brown@ntu.ac.uk; phone 44115 848-3184; fax 44115 848-6636; www.ntu.ac.uk/science_technology/

\section{INTRODUCTION}

\subsection{Nematic liquid crystal phase gratings}

Switchable liquid crystal diffraction gratings have applications for optical beamsteering in optical fibre based telecommunication systems ${ }^{[1,2]}$. A number of devices have been demonstrated based on the switching of a layer of planar nematic liquid crystal to produce a periodic spatially varying phase profile ${ }^{[3-8]}$. When no voltages are applied the molecular director of the nematic liquid crystal through the layer is oriented along a direction pre-determined by unidirectional rubbing of the substrates. Switching is produced via a striped electrode array on one substrate opposite to a continuous earth plane on the opposite substrate. By applying a suitable periodically repeating pattern of voltages a blazed or sawtooth phase profile can be produced which allows efficient beamsteering for electromagnetic waves that are polarised along the surface rubbing direction. Under ideal circumstances there should be abrupt transitions between the linearly varying regions in the sawtooth phase profile, referred to as "fly-back" regions. The liquid crystal is unable to reproduce these abrupt transitions due to the inherent elasticity of the medium. This property, coupled with the effects of fringing electric fields at electrode edges, leads to smooth transitions in the phase profile and the widening of the flyback regions.

\subsection{Device details}

In the current work the switching of the nematic liquid crystal in response to fringing electric fields at the edges of striped interdigitated electrodes has been used to study flexoelectric polarisation effects in a nematic liquid crystal material. The device geometry is shown in Fig. 1. The nematic liquid crystal layer is confined in layer of thickness $d=17.2 \mu \mathrm{m}$ in the $\mathrm{x}-\mathrm{y}$ plane. The two glass substrates that confine the layer are coated with indium tin oxide electrodes (conductivity $50 \Omega$ /square) and a polymer alignment layer (Polyvinylalcohol, PVA). The electrode on the upper substrate $(z=d)$ is continuous and was held at earth potential throughout the experiment. The electrode on the lower plate $(z=0)$ has been etched to form an array of parallel stripes. The 2 dimensional electrode pattern in the $x-y$ plane was designed so 
that different potentials could be applied to alternate electrodes, as shown in Fig. 1. The photolithographic and etching process that was used to fabricate the interdigitated electrodes gave electrodes widths (37 $\mu \mathrm{m})$ that were slightly narrower than the gaps between the electrodes $(43 \mu \mathrm{m})$. The PVA polymer layer was uni-directionally rubbed in the positive $\mathrm{x}$ direction on the lower plate and the negative $\mathrm{x}$ direction on the upper plate. This gave planar alignment with a slight tilt angle throughout the layer equal to the pretilt of the nematic director at the PVA substrates. The device was capillary filled with the commercial liquid crystal material E7 ${ }^{[9]}$. This material is has a relatively high dielectric anisotropy $\left(\Delta \varepsilon=13.9\right.$ at $\left.22{ }^{\circ} \mathrm{C}\right)$ and it is known to be susceptible to ionic contamination ${ }^{[10]}$.

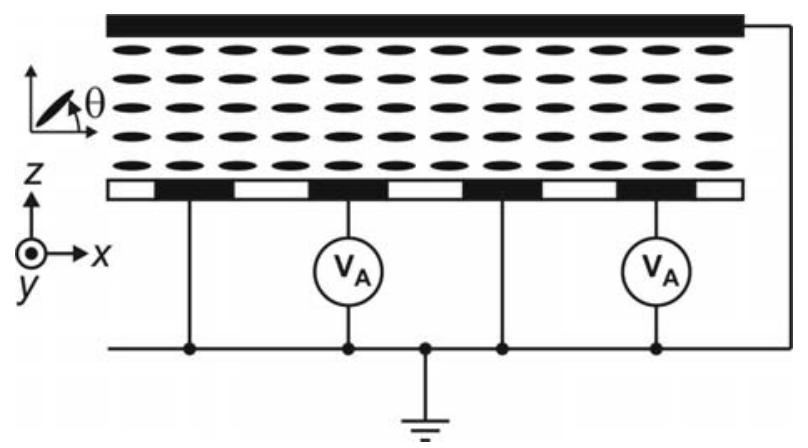

Fig. 1. Schematic diagram of the device geometry. The filled black rectangles show the positions of indium tin oxide electrodes. The striped electrodes on the lower substrate were fabricated in an interdigitated geometry so that alternate electrodes could be differently biased.

\section{MEASUREMENTS USING A MACH-ZEHNDER INTERFEROMETER}

\subsection{Time dependent phase profile}

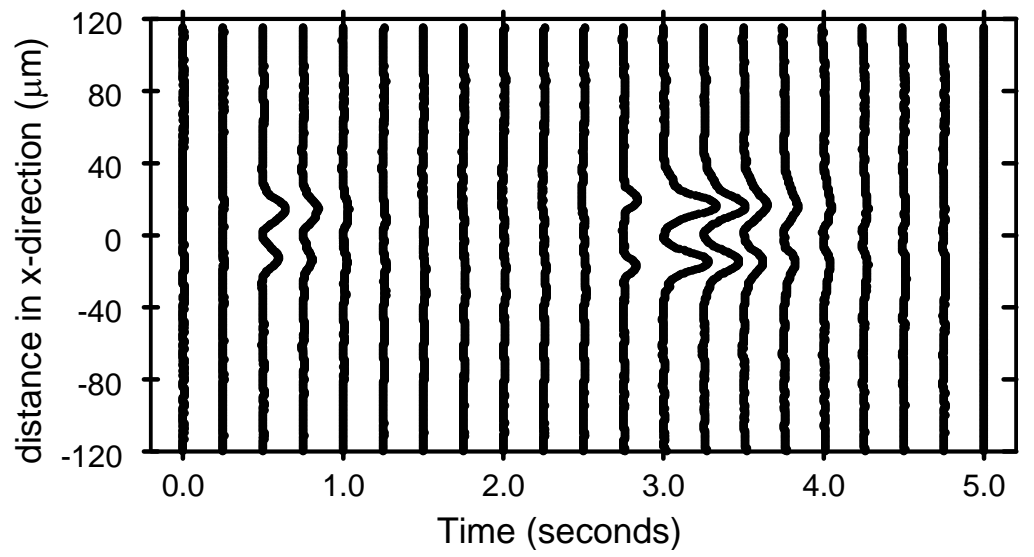

Fig. 2. Displacement of tilt fringes observed when the device of Fig. 1 was placed an arm of a Mach-Zehnder interferometer with the fringes parallel to the $\mathrm{x}$-direction. The voltage was negative $\left(V_{A}=-1.17 \mathrm{~V}\right)$ during the time interval $0.0<t<2.5 \mathrm{sec}$, and positive $\left(V_{A}=+1.17 \mathrm{~V}\right)$ during the time interval $2.5<t<5.0 \mathrm{sec}$.

The device was placed in one arm of a Mach-Zehnder interferometer that was illuminated with a He-Ne laser light with wavelength $632.8 \mathrm{~nm}$ and polarization in the x-direction. One of the mirrors of the interferometer was tilted so that sinusoidal fringes were superimposed onto an image of the device formed by a relay lens-pair ${ }^{[11]}$. The sinusoidal intensity variation of the fringes occurred in the $y$-direction and so each individual fringe ran parallel to the $\mathrm{x}$-axis. Under these conditions the position of the fringe is displaced when the nematic n-director in the layer reorients in the x-y plane 
to a non-zero angle $\theta(x, z, t)$ with the $x$-direction. This occurs because the position of the fringes are displaced when the optical path length in one arm of the interferometer changes. The material E7 is a positive uniaxial medium and so this reorientation of the n-director reduces the refractive index experienced by an electromagnetic wave travelling in the $\mathrm{z}$ direction and polarized in the $\mathrm{x}$-direction. A fringe is displaced in position by one fringe spacing when the total optical path length through the nematic liquid crystal layer $k \cdot d$ is equal to $2 \pi$ radians. The wavevector is given by $k=2 \pi n_{\text {eff }}(\mathrm{x}) / \lambda_{\mathrm{O}}$ where $n_{\text {eff }}$ equals the average refractive index for a $z$-section through the layer at the position $x$.

Fig 2 shows the shapes of the fringes at different times. Each fringe shown on the figure is an average of 6 adjacent fringes that were measured in the interferometer. The intersection of each fringe with the x-axis in Fig. 1 corresponds to the time at which it was recorded. Each of the fringes shown in Fig. 2. spans a region of the device of width $240 \mu \mathrm{m}$ in the x-direction. For clarity, the amplitude of each fringe has been multiplied by a factor of two so the distance between two fringes on the $\mathrm{x}$-axis corresponds to the distance between adjacent fringes in the pattern observed from the interfereometer. The centre electrode in Fig. 1 was biased at potential $V_{A}(-20 \mu \mathrm{m}<x<20 \mu \mathrm{m})$ and the adjacent electrodes $(-100 \mu \mathrm{m}<x<-80 \mu \mathrm{m}$ and $100 \mu \mathrm{m}<x<100 \mu \mathrm{m})$ were held at earth potential.

The fringes in Fig. 2 are shown every $0.25 \mathrm{sec}$ for times between $t=0.0 \mathrm{sec}$ and $t=5.0 \mathrm{sec}$. During the measurement a squarewave voltage of frequency $0.2 \mathrm{~Hz}$ and amplitude $V_{A}=1.17 \mathrm{~V}$ was applied to alternate electrodes in the striped interdigitated electrode array. The voltage was negative $\left(V_{A}=-1.17\right)$ during the time interval $0.0<t<2.5 \mathrm{sec}$, and positive $\left(V_{A}=+1.17 \mathrm{~V}\right)$ during the time interval $2.5<t<5.0 \mathrm{sec}$.

The two positions in Fig. 2 where displacement of the fringes occur are at $x=-15 \mu \mathrm{m}$ and $x=+15 \mu \mathrm{m}$. These regions correspond to the edges of the biased centre electrode and are due to distortions in the nematic director alignment associated with the fringing electric fields near to the electrode edges. The evolution of the fringe displacement profiles with time in Fig. 2 has been discussed in detail in a previous publication ${ }^{[12]}$. The displacements increase in amplitude as a function of time after the changes in polarity of the voltage $V_{A}$ which occur at $t=0.0 \mathrm{sec}$ and $t=2.5 \mathrm{sec}$. The displacements reach a peak between $0.25 \mathrm{sec}$ and $0.5 \mathrm{sec}$ after the change in polarity and then decay. The decay has been attributed to the motion of mobile ionic species in the nematic material which move to shield the applied voltage ${ }^{[13]}$. The peaks in the displacement are larger for the positive applied voltage than the negative applied voltage and this asymmetry is due to the flexoelectric polarisation in the nematic liquid crystal material ${ }^{[14,15]}$. For both polarities the peak at $x=+15 \mu \mathrm{m}$ is slightly larger than the peak at $x=-15 \mu \mathrm{m}$, which can be explained by the antiparallel alignment direction coupled with the influence of the small surface pretilt ${ }^{[12]}$.

\subsection{Time dependent $2 \mathrm{~d}$ nematic director configuration}

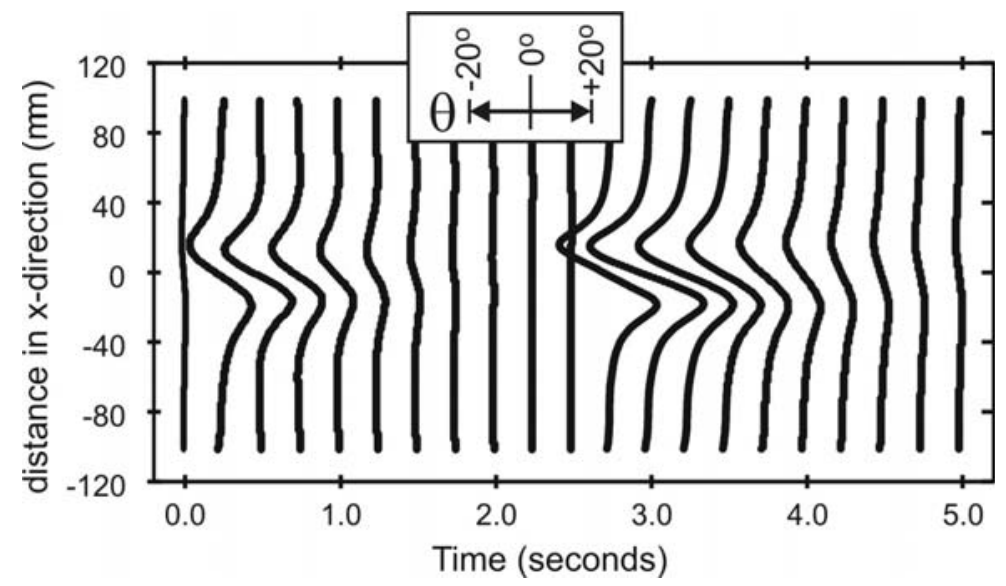

Fig. 3. The calculated director angle along a horizontal section through the device shown in Fig. 1 at the position $z=d / 2$.

The evolution of the fringe displacement profiles with time in Fig. 2 has been fitted using nematic continuum theory in a previous publication ${ }^{[12]}$. The following materials parameters were taken from literature and auxiliary measurements : splay and bend elastic constants, $10.5 \mathrm{pN}$ and $15.2 \mathrm{pN}$ respectively; permittivity parallel and perpendicular to the n- 
director, 19.1 and 5.2 respectively; and refractive index parallel and perpendicular to the n-director 1.735 and 1.518 respectively. The empty cell thickness was measured as $17.2 \mu \mathrm{m}$ using interference fringes in monochromatic light. In order to account for the migration and shielding of mobile ionic species a time dependent exponentially decaying voltage waveform $V_{A}(t)= \pm V_{O} \exp (-t / \tau)$ was used for each polarity of the applied voltage. The following values were obtained from the fit, as reported in reference [12] : surface pretilt $0.4^{\circ}$; viscosity associated with rotation of the nematic director $0.18 \mathrm{Nsm}^{-2}$; voltage paraneters $V_{O}=1.89 \mathrm{~V}$ and $\tau=0.80 \mathrm{sec}$; and the sum of the flexoelectric coefficients was $\left(e_{1}+e_{3}\right)=(14 \pm 1) \mathrm{pC} \mathrm{m}^{-1}$.

The time dependent director profile $\theta(x, z, t)$ throughout the nematic liquid crystal layer in response to the exponentially decaying voltage waveform was calculated as part of the fitting process in reference [12] in order to obtain these parameters. Fig. 3 shows the director angle along a horizontal section through the cell of Fig. 1 at the position $z=d / 2$ for the same times during the switching cycle as for Fig. 2. Note that the director tilt is in opposite directions at opposite sides of the centre electrode where the voltage $V_{A}$ was applied. This occurs because the dominant switching mechanism of the nematic liquid crystal is the dielectric coupling to the fringing electric fields. The presence of the flexoelectric polarization in the distorted nematic liquid crystal in these regions causes an enhancement in the reorientation (higher tilt angles) for positive $V_{A}$ but lower reorientation (lower tilt angles) for negative $V_{A}$. The profiles in Fig. 3 will be used for the interpretation of the results from the optical diffraction measurements that are reported in the current article for the first time.

\section{OPTICAL DIFFRACTION MEASUREMENTS}

\subsection{Static optical diffraction patterns}

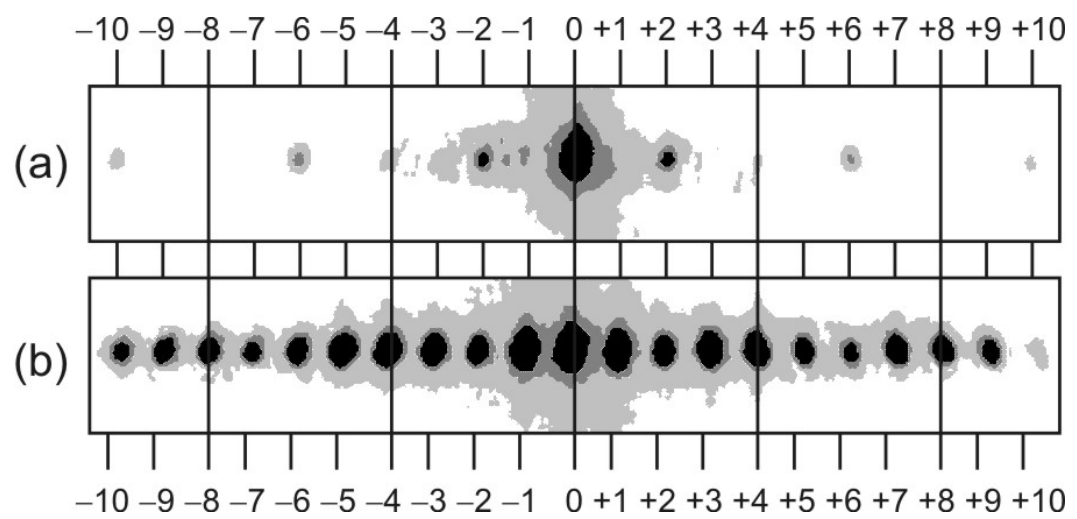

Fig. 4. Far field diffraction pattern produced by the device geometry shown in Fig. 1 : (a) No applied voltage and, (b) a.c. sinusoidal voltage with frequency $1.0 \mathrm{kHz}$ and amplitude $V_{A}=1.17$ Vrms.

Static far field diffraction patterns are shown in Fig. 4 when the device was illuminated with collimated He-Ne laser light of wavelength $632.8 \mathrm{~nm}$. In Fig. 4(a) all of the electrodes were connected to earth potential. The diffraction pattern is due to the indium tin oxide striped electrodes on the substrate at $\mathrm{z}=0$. The diffraction orders labeled as -2 and +2 correspond to the electrode period of $80 \mu \mathrm{m}$ i.e. $37 \mu \mathrm{m}$ electrodes plus $43 \mu \mathrm{m}$ gaps. The periodic striped electrodes act as a square-wave transmission grating and so only the even diffraction orders $( \pm 2, \pm 6, \pm 10)$ appear in Fig. 4(a) and the odd orders are missing.

A sinewave voltage with frequency $1.0 \mathrm{kHz}$ and amplitude $V_{A}=1.17 \mathrm{Vrms}$ was applied to produce the far field diffraction pattern shown in Fig. 4(b). A reorientation of the nematic liquid occurs in the edge regions of the alternate electrodes to which the voltage is applied. The laser light was polarized in the x-direction and so it experienced the spatially periodic lowering of the refractive index associated with the reorientation regions. This gives rise to the -1 and +1 first diffraction orders corresponding to twice the electrode period, i.e. $160 \mu \mathrm{m}$. A range of higher order diffraction 
spots occurs in Fig. 4(b) and the intensity envelope of the diffraction orders is determined, to the first approximation, from the profile of the average refractive index due to the distortion in the nematic as a function of the x coordinate.

\subsection{Time dependent optical diffraction patterns}

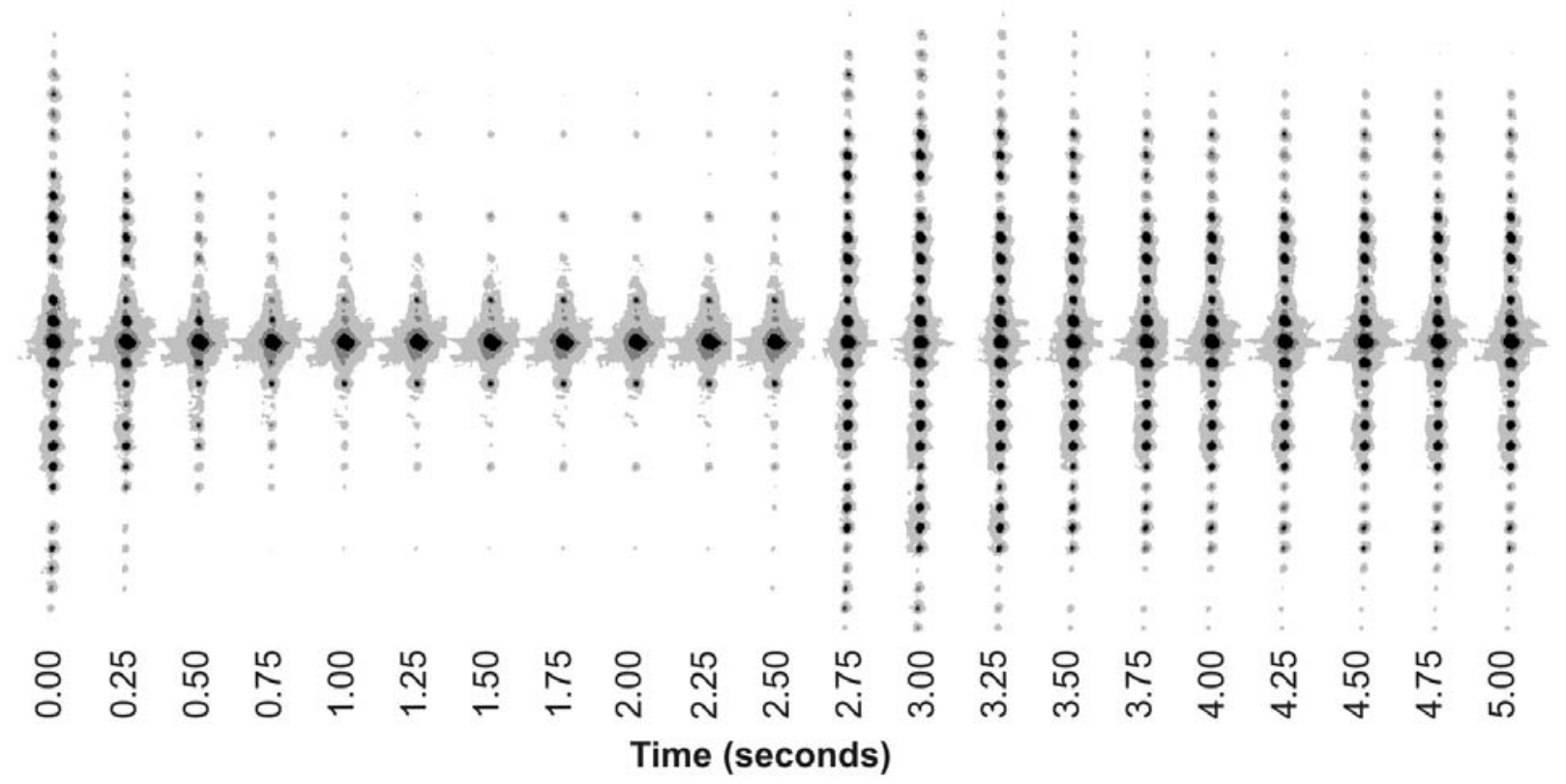

Fig. 5. Far field diffraction pattern produced by the device geometry shown in Fig. 1 as a function of time. The voltage was negative $\left(V_{A}=-1.17 \mathrm{~V}\right)$ during the time interval $0.0<t<2.5 \mathrm{sec}$, and positive $\left(V_{A}=+1.17 \mathrm{~V}\right)$ during the time interval $2.5<t<5.0 \mathrm{sec}$.

The far field optical diffraction patterns were next measured during the application of a squarewave voltage of frequency $0.2 \mathrm{~Hz}$ and amplitude $V_{A}=1.17 \mathrm{~V}$ to alternate electrodes in the striped interdigitated electrode array. The resultant diffraction patterns in Fig. 4 are shown at 0.25 sec intervals and the voltage was negative $\left(V_{A}=-1.17\right)$ during the time interval $0.0<t<2.5 \mathrm{sec}$ and positive $\left(V_{A}=+1.17 \mathrm{~V}\right)$ during the time interval $2.5<t<5.0 \mathrm{sec}$ so that a direct comparison with Figs. 2 and 3 can be made. The images have been digitized to show 16 grey-levels and the darker color corresponds to a diffraction spot having higher intensity.

Immediately after the application of the positive voltage ( $t>2.50 \mathrm{sec}$ ) a series of strong diffraction spots appear up to order \pm 15 . The highest orders reduce in intensity within $1.25 \mathrm{sec}$ but the lower orders persist throughout the duration of the positive voltage. After the change in polarity to the negative voltage ( $t>0 \mathrm{sec}$ in Fig. 5 ) the diffracted orders up to \pm 8 are strong but there is a fast decay within $0.25-0.5 \mathrm{sec}$. By $1.25 \mathrm{sec}$ after the change to the negative polarity the diffraction pattern is similar to the diffraction pattern in Fig. 4(a) which is solely due to the diffraction from the electrodes. These observations are consistent with the director tilt profiles shown in Fig. 3 where there is a larger tilt after the application of the positive pulse then after the application of the negative pulse, and there is still a significant tilt distortion across the nematic layer $2.5 \mathrm{sec}$ after the positive pulse which continues to diffract light.

The time dependent intensity of the first 4 diffracted orders is shown in Fig. 6. The labeling system is the same as that shown in Fig. 4, i.e. the \pm 1 orders correspond to diffraction from a spatial period of $160 \mu \mathrm{m}$. Fig. 6(a) shows the time dependent intensities of the zero (solid line), \pm 1 (squares) and \pm 2 (diamonds) orders and Fig. 6(b) shows the time dependent intensities of the \pm 3 (squares) and \pm 4 (diamonds) orders, both with a logarithmic intensity scale. For both of the graphs the filled symbols show data for the + orders and the empty symbols for the - diffracted orders.

For the $\pm 1, \pm 3$ and \pm 4 orders shown there is a peak in the intensity after a change in polarity of the applied voltage and this is followed by a decay of the intensity. This decay is steeper after the change from positive to negative polarity. It was discussed in section 2.2 above that although the applied voltage was a $0.2 \mathrm{kHz}$ square-wave with a constant 
amplitude of $1.17 \mathrm{~V}$, the data from the measurements performed on the Mach-Zehnder interferometer could only be fitted using nematic continuum theory if the voltage actually experienced by the liquid crystal layer was assumed to be exponentially decaying in each half of the waveform cycle due to the shielding effect of mobile ionic charges in the liquid crystal medium. The same effect is observed in the optical diffraction measurements.

The \pm 2 orders show an increase in intensity with time after the initial decay. It should be noted, however, that the even orders are influenced by diffraction from the periodic striped electrodes that have a slightly lower transmission than the uncoated glass substrate.

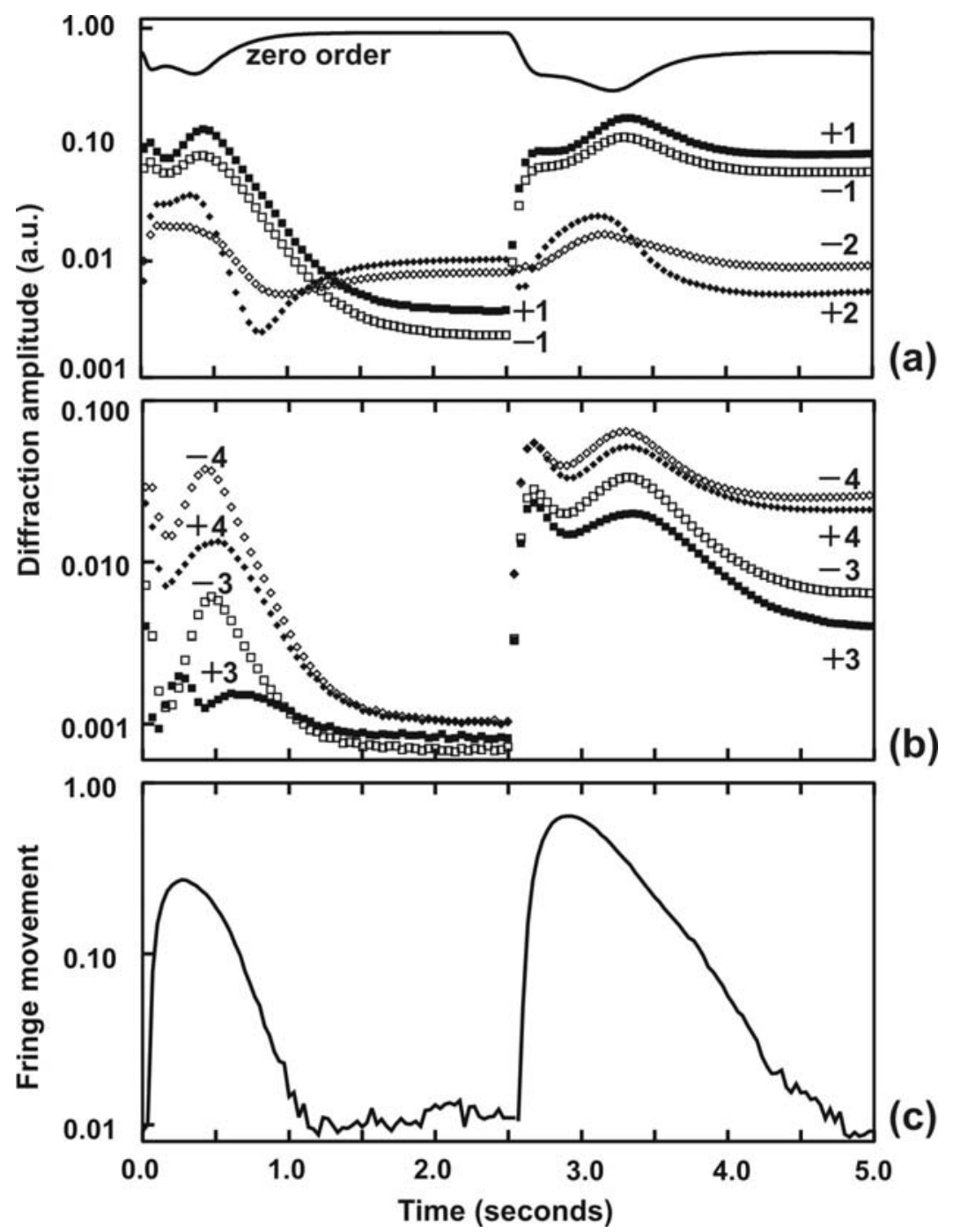

Fig. 6. (a) and (b) show the time dependent intensity of different orders in the far field diffraction pattern shown in Fig. 5. (c) shows the displacement of fringes in an Mach-Zehnder interferometer at $\mathrm{x}=15 \mu \mathrm{m}$ as a function of time. This corresponds to a horizontal cross-section through Fig. 2. The voltage was negative $\left(V_{A}=-1.17 \mathrm{~V}\right)$ during the time interval $0.0<t<2.5 \mathrm{sec}$, and positive $\left(V_{A}=+1.17 \mathrm{~V}\right)$ during the time interval $2.5<t<5.0 \mathrm{sec}$.

\section{DISCUSSION AND CONCLUSIONS}

The influence of the flexoelectric polarization on the d.c. switching of a nematic liquid crystal phase grating has been investigated by observing the far field optical diffraction pattern. The diffracted orders show an asymmetry in intensity in response to positive and negative applied voltages due to the presence of the flexoelectric polarisation. In general, the 
intensities of the diffracted spots decay with time due the voltage shielding of mobile ionic species. This decay is faster after a negative voltage has been applied than after a positive voltage has been applied. There is also an asymmetry in the time dependent intensities of the + and - diffracted orders since the surface pretilt at the alignment layers is in opposite directions on the opposite substrates.

The results have been compared with measurements on the same device of the displacement of tilt fringes in a MachZehnder interferometer ${ }^{[12]}$. Fig. 6(c) shows the logarithm of the fringe displacement measured using the Mach-Zehnder interferometer at the position $\mathrm{x}=15 \mu \mathrm{m}$ on the device. This shows a similar behavior as a function of time particularly to the \pm 3 diffraction orders. However, the full decay after the applied d.c. voltage with negative polarity cannot be measured because of random noise. The fringe displacement can only be measured to around 1-2\% of the separation between adjacent fringes because the resolution of the interferometer is limited by the magnification optics and even a well isolated instrument is susceptible to environmental vibrations.

\section{REFERENCES}

[1] Crossland, W.A. and Wilkinson, T.D., "Nondisplay Applications of Liquid Crystals”, [Handbook of Liquid Crystals], Wiley-VCH, Weinhem (1998)

[2] Kriezis E.E. and Brown C.V., "Liquid Crystal Diffractive Optical Elements”, [Handbook of Organic Electronics and Photonics Volume 2], American Scientific Publishers, 235-299 (2008)

[3] Efron, U. Apter, B. Bahat-Treidel E. , Journal of the Optical Society of America 21, 1996 (2004)

[4] Apter B., Efron U., and Bahat-Treidel E., Applied Optics 43, 11 (2004)

[5] Hällstig E., Stigwall J., Martin T., Sjöqvist L. and Lindgren M., Journal of Modern Optics 51, 1233 (2004)

[6] Resler D.P., Hobb D.S., Sharp R.C., Friedman L.J. and Dorschner T.A., Optics Letters 21, 689 (1996)

[7] Tanone A., Zhang Z., Uang C.-M and Yu F.T.S., Microwave and Optical Technology Letters 7, 285 (1994)

[8] Matic R.M., Proc. SPIE 2120, 194 (1994)

[9] E7 room temperature nematic liquid crystal, Liquid Crystals Division, Merck KGaA, 64271 Darmstadt, Germany.

[10] Heilmeier G.H., Zanoni L.A., and Barton L.A., Appl. Phys. Lett. 13, 46 (1968)

[11] Martin-Badosa E., Carnicer A., Juvells I. and Vallmitjana S., Meas. Sci. Tech. 8, 764 (1997)

[12] C.Trabi, C.V. Brown, A.A.T. Smith and N.J. Mottram, Applied Physics Letters 92, 223509 (2008)

[13] M. Scalerandi, P. Pagliusi, G. Cipparrone and G. Barbero, Phys, Rev. E. 69, 051708 (2004)

[14] Meyer R.B., Phys. Rev. Lett. 22, 918 (1969)

[15] DeGennes P.G. and Prost J., [The Physics of Liquid Crystals], 2nd Edition, Oxford Science Publications, pp 135139 (1995)

Camera ready version of the paper that was subsequently published as:

"Flexoelectric polarisation effects in nematic liquid crystal phase gratings." - art. no. 70500R

Trabi CL, Smith AAT, Mottram NJ and Brown CV

Proc. SPIE Liquid Crystal Xii, Vol 7050, p R500 (2008) 